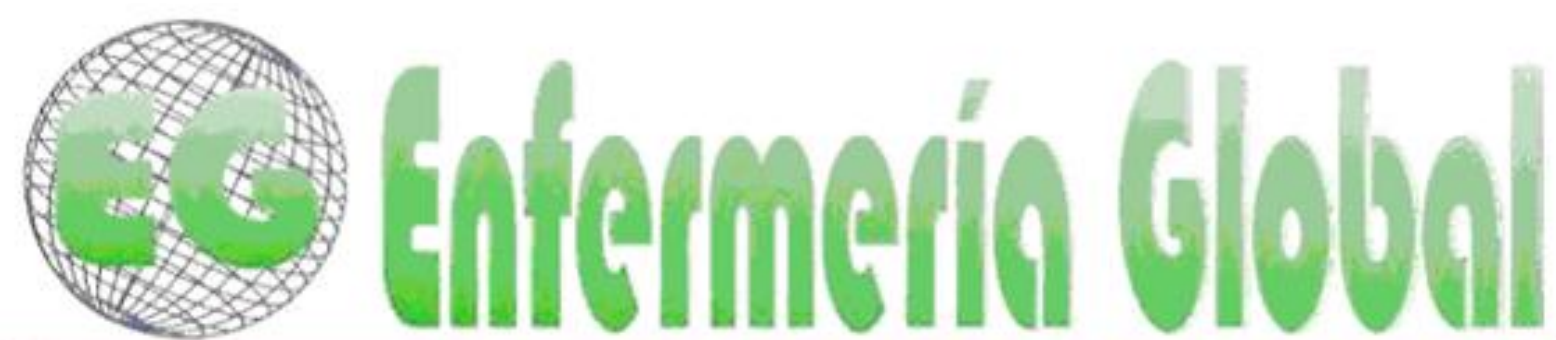

ISSN 1695-6141

Revista electrónica trimestral de Enfermería

$N^{\circ} 44$

Octubre 2016

www.um.es/egloball

\title{
CLÍNICA
}

\section{Prevalencia de la Lactancia Materna en Huelva, duración y factores relacionados}

Prevalence of Breastfeeding in Huelva, duration and related factors

\section{*Burguillo, María del Pilar Tierra ${ }^{* *}$ Campos, Zahira Villa ***Marín Sánchez, Carmen ${ }^{* * * *}$ Pedregal González, Miguel ${ }^{* * * *}$ Muñoz Soriano, $\mathrm{M}^{\mathrm{a}}$ Isabel ******Álvarez Palacios, $\mathrm{M}^{a}$ del Pino}

\begin{abstract}
*Unidad de Pediatría, Hospital Juan Ramón Jiménez, Complejo Universitario de Huelva, Servicio Andaluz de Salud. E-mail: pilartierra@hotmail.com **Mutua Cesma, Huelva. ***Centro de Salud El Molino, Huelva. ****Unidad Docente de Medicina Familiar y Comunitaria. Distrito Huelva-Costa. ${ }^{* * * *}$ Capri Community Health Centre, Kelowna, Canadá.******Centro de Salud Isla Chica, Huelva, España.
\end{abstract}

Palabras clave: Lactancia; Lactancia Materna; Prevalencia; Centros de Salud

Keywords: Breast Feeding; Prevalence; Health Centers.

\section{RESUMEN}

Objetivos: Conocer la prevalencia y duración de la Lactancia Materna (LM), así como la relación con las variables sociodemográficas, obstétricas y de educación materno infantil.

Diseño: Estudio observacional descriptivo. Emplazamiento: siete centros de salud de Huelva capital. Participantes: 268 mujeres con niños de 1 a 2 años. La selección se hizo consecutiva, por orden de llegada. Mediciones principales: prevalencia y duración de LM, características sociodemográficas, características perinatales y alimentación complementaria.

Resultados: Iniciaron la LM exclusiva un 52,9 \% (IC:46,99-58,91). A los seis meses permanecen un 19,7\% (IC:15,33-24,87). Un 63,4\% (IC:57,54-69,04) comienzan lactancia materna total (exclusiva o mixta), un 47,7\% (IC:41,82-53,75) continúa con ella a los 3 meses y un 26,1\% (IC:21,12-31,63) a los 6 meses. La LM está relacionada con: la formación académica de la madre, el número de hijos, asistir a los talleres de preparación al parto, número de sesiones, nivel de satisfacción con respecto a la educación materno infantil, haber solicitado ayuda sobre LM, conocer el grupo de apoyo a la lactancia y haber asistido al mismo.

Conclusiones: La prevalencia de LM es baja con respecto a lo esperado por los datos nacionales, la duración de la misma se aparta de las recomendaciones de la OMS y el nivel educativo de la madre 
favorece la prevalencia y duración de la LM. Los profesionales de la salud debemos continuar con su promoción y apoyo.

\section{ABSTRACT}

Objective: To know the prevalence and duration of the breastfeeding, as well as the relation with the sociodemographic, obstetric and child and maternal education factors.

Design: descriptive observational study. Site: seven health centers of Huelva city. Participants: 268 mothers of 1-2 y.o. children. The selection was made straight, in order of arrival. Main measurements: prevalence and duration of the breastfeeding, sociodemographic characteristics, perinatal characteristics and complementary feeding.

Results: The $52,9 \%(\mathrm{Cl}: 46,99-58,91)$ of the sample initiated the exclusive breastfeeding. Six months later remained the $19,7 \%(\mathrm{Cl}: 15,33-24,87)$. The $63,4 \%(\mathrm{Cl}: 57,54-69,04)$ initiated the total breastfeeding, 47,7\% (Cl:41,82-53,75) continue with it 3 months later and the $26,1 \%(\mathrm{Cl}: 21,12-31,63) 6$ months later. The breastfeeding is related with: the academic education of the mother, the number of children, to attend the workshops childbirth preparation, number of sessions, level of satisfaction with maternal and child education, to have requested assistance on breastfeeding, to know the breastfeeding support group and to have atended to it.

Conclusions: The prevalence of the breastfeeding is less concerning than expected by the national data, the length of it is far from the recommendations of the WHO and the educational level of the mother, favors the prevalence and duration of breastfeeding. Health professionals must continue with their promotion and support.

\section{INTRODUCCIÓN}

La leche materna tiene las características necesarias para proporcionar toda la energía y los nutrientes que los lactantes necesitan durante sus primeros meses de vida ${ }^{(1)}$. Son numerosos los estudios que demuestran la valía de la misma ${ }^{(2)}$ y defienden una lactancia materna exclusiva (LME) hasta los 6 meses, como desde el año 2001 promueve la OMS ${ }^{(3)}$. Esta organización junto con UNICEF recomienda iniciar la lactancia materna (LM) durante la primera hora de vida, evitar el uso de biberones, tetinas o chupetes y proporcionarla LME a demanda ${ }^{(1)}$.

Lo que en la gran mayoría de los países no tiene más misterio que el de ofrecer el pecho al bebé cada vez que éste lo demande, en el caso de países como España la LM es abandonada en muchas ocasiones por motivos fácilmente evitables ${ }^{(4)}$. Hay estudios que evidencian que un asesoramiento adecuado al respecto, aumenta la duración de la misma. Este asesoramiento puede verse materializado de muchas formas, mediante el papel de: una doula ${ }^{(5)}$, un servicio telefónico especializado ${ }^{(6)} \ldots$

Pero en nuestro país y más concretamente en el contexto similar donde se desarrolla el estudio, Huelva, está comprobado que con una buena actuación por parte del personal sanitario se conseguiría el objetivo esperado ${ }^{(7)}$. Este asesoramiento se puede encontrar en los centros asistenciales: hospitales y centros de salud. A su vez en la asociación de apoyo a la lactancia.

Según la Encuesta Nacional de Salud 2011-2012, la prevalencia de la lactancia materna total (LMT) a las 6 semanas, 3 meses y 6 meses es de 72,40\%,66,6\% y $46,90 \%$ respectivamente ${ }^{(8)}$. Los estudios que analizan la relación de la LM con otras variables muestran resultados a favor de la educación para la salud en LM como principal promotor de la misma, tanto en la prevalencia como duración ${ }^{(9-13)}$.Ofrecen resultados favorecedores en relación a la edad de las madres, donde las de mayor 
edad lactan con más duración que las más jóvenes ${ }^{(11,14)}$. Hay estudios que demuestran que los partos eutócicos tienen relación estadísticamente significativa con la LM ${ }^{(11)}$, otros que confirman esta relación con la formación académica de las madres ${ }^{(11,14)}$ y por otro lado, la variable trabajo remunerado presenta resultados diferentes según la literatura científica revisada ${ }^{(4)}$. Este estudio tiene como objetivo conocer la prevalencia y duración de la LM en Huelva capital y las variables relacionadas con la misma.

\section{METODOLOGÍA}

\section{Objetivos}

- Conocer la prevalencia y duración de la Lactancia Materna en Huelva capital.

- Identificar las variables que presenten relación con la prevalencia y duración de la Lactancia Materna.

Diseño

Estudio observacional descriptivo.

Ámbito de estudio

Los siete centros de atención primaria del distrito Huelva-Costa que se encuentran situados dentro del núcleo urbano de la ciudad.

Periodo de estudio

Mayo de 2012 a enero 2014

Población de estudio

Mujeres pertenecientes a los centros de salud de Huelva con hijos con edades comprendidas entre los 12 y los 24 meses.

\section{Criterios de inclusión}

- Tener un hijo con edad comprendida entre los 12 y 24 meses.

- Pertenecer a alguno de los siete centros de salud de Huelva.

- Aceptar el consentimiento informado necesario para la realización de la encuesta.

Tamaño de la muestra

Para una prevalencia del 50\%, una precisión del $6 \%$ y una confianza del $95 \%$. El tamaño de la muestra final fue de 268 mujeres.

\section{Variables}

a) Variables principales:

LM y duración de la LM en meses, desde el nacimiento.

La duración se valoró teniendo en cuenta el tipo de lactancia en el momento de la 
encuesta y la fecha de abandono en los casos que había finalizado. Se diferenció entre LME y LMT, donde se incluyó todo el tiempo que el niño estuvo recibiendo alguna toma de pecho, aunque ésta estuviera acompañada por leche artificial.

b) Variables secundarias:

b.1. Datos sociodemográficos de la madre:

- Estado civil: soltera, casada o divorciada.

- Formación académica: sin estudios, EGB/ESO, Bachiller/módulo/FP o universitaria.

- Situación laboral: empleada o desempleada.

- Número de hijos.

- Con quién convive: con su pareja, sola, con sus padres u otro.

b.2. Datos perinatales:

- Edad de la madre en el momento del parto.

- Tipo de parto: natural o quirúrgico.

- Principales fuentes de información durante el embarazo: experiencia personal, familia, pediatra, matrona e Internet.

- Haber asistido a los talleres de preparación al parto y el número de sesiones.

- Haber solicitado ayuda sobre LM.

- Nivel de satisfacción sobre la educación materno infantile recibida. Se ha usado una escala del 1 al 10, donde el 1 es el valor mínimo y el 10 el máximo.

- Conocer la asociación de apoyo a la lactancia.

- Haber asistido a alguna reunión de esta asociación.

- Haber realizado ejercicio físico durante el embarazo.

- Haber realizado actividades postparto con su bebé.

b.3. Datos del niño:

- Edad de inicio de la lactancia artificial, en meses.

- Edad de inicio de la alimentación complementaria, en meses.

- Haber asistido a un centro de educación infantil.

Recogida de datos

Para la recogida de datos se realizó el diseño y se validó un cuestionario, mediante una prueba de jueces y un pilotaje. Los cuestionarios se han administrado en las salas de espera de los centros de salud, por parte del personal de enfermería que dirige este estudio.

Análisis de datos

El programa informático que se utilizó para realizar el análisis estadístico fue el SPSS. Para realizar el análisis descriptivo de las variables cuantitativas se halló la media y la desviación típica. Para las cualitativas, el porcentaje.

El test estadístico específico para comprobar la normalidad que se ha usado es el Shapiro-Wilk.

Para hallar la homogeneidad de las varianzas se ha usado el test de Levene. 
Para las comparaciones de dos grupos en una variable cuantitativa, se ha usado la $t$ de Student y para más de dos grupos, el ANOVA de un factor. Para el análisis de variables cualitativas el Chi Cuadrado de Pearson.

A su vez, se han usado tests no Paramétricos para aquellos grupos que no cumplían las condiciones de normalidad: Kruskal-Wallis y Jonckheere-Terpstra, pruebas no paramétricas de la ANOVA, el último, de tendencia lineal y el U Mann-Witney.

Se realizó un análisis de regresión logística binaria cuya variable dependiente fue la LM y la relación de las variables que de manera independiente influyen en la misma. Y una regresión lineal múltiple para la variable dependiente duración de la LM.

\section{Aspectos éticos}

A lo largo del estudio se han respetado los principios básicos de la declaración de Helsinki y el código de ética médica de Núremberg.

Antes de llevar a cabo el estudio se obtuvo la aprobación por parte del comité de Ética e Investigación provincial. Y previo a la recogida de datos en los centros de salud, el consentimiento por parte de la directora del distrito Huelva Costa de la provincia de Huelva y de los directores de cada uno de ellos. A cada una de las mujeres se les explicó el fin de la misma y se le solicitó un consentimiento informado. Todas y cada una de las mujeres que participaron sabían que estaban colaborando con un trabajo de investigación. Se ha respetado su anonimato y la confidencialidad de los datos.

\section{RESULTADOS}

La muestra estaba formada por 268 mujeres con edades comprendidas entre los 17 y los 44 años, y la media de edad es de 31,29 años (DT 5,16). El 70,9\% (190) estaban casadas y el $86,6 \%$ (232) vivían con sus parejas. El 74,8\% (237) eran multíparas y la media del número de hijos fue de 1,7 (DT 0,8). El 68,6\% (183) poseían estudios medios y el 33,2\% (89) universitarios. El 47,7\% (128) trabajaba de manera remunerada. La muestra presentaba un 7,1 (DT 1,7) sobre 10 de satisfacción sobre educación materno infantil recibida.

Un 52,9\% de la muestra refería haber dado LME. El 47,7\% lactó a sus bebés hasta las 6 semanas, el 40,6\% hasta los 3 meses y el 19,7\% a los 6 meses, 19,7\% (tabla 1).

Tabla 1. Prevalencia de la lactancia materna exclusiva y total, Huelva, España, 2013.

\begin{tabular}{|c|c|c|c|c|c|c|}
\hline \multirow{2}{*}{$\begin{array}{c}\text { Edad } \\
\text { del niño }\end{array}$} & \multicolumn{3}{|c|}{ Lactancia Materna Exclusiva } & \multicolumn{3}{|c|}{ Lactancia Materna Total } \\
\hline & $\mathbf{n}$ & prevalencia $(\%)$ & IC del $95 \%$ & $\mathbf{n}$ & prevalencia $(\%)$ & IC del $95 \%$ \\
\hline 0 meses & 142 & 52,9 & $46,99-58,91$ & 170 & 63,4 & $57,54-69,04$ \\
\hline 6 semanas & 128 & 47,7 & $41,82-53,75$ & 147 & 54,8 & $48,86-60,91$ \\
\hline 3 meses & 109 & 40,7 & $34,91-46,64$ & 128 & 47,8 & $41,82-53,75$ \\
\hline 6 meses & 53 & 19,8 & $15,33-24,87$ & 70 & 26,1 & $21,12-31,63$ \\
\hline
\end{tabular}

La formación académica de la madre influyó en la prevalencia de la $\operatorname{LM}(p=0,045)$. De esta manera, el porcentaje de madres que dan el pecho aumenta con la formación académica de éstas (tabla 2). 
Tabla 2.- Relación entre la LM y la formación académica de las madres.

\begin{tabular}{|c|c|c|c|}
\hline Formación Académica & LM & Lactancia artificial & n \\
\hline Sin estudios & $6(46,2 \%)$ & $7(53,8 \%)$ & 13 \\
\hline EGB/ESO & $40(56,3 \%)$ & $31(43,7 \%)$ & 71 \\
\hline Bachiller/Modulo/FP & $63(66,3 \%)$ & $32(33,7 \%)$ & 95 \\
\hline Universitarios & $61(68,5 \%)$ & $28(31,5 \%)$ & 89 \\
\hline Total & $170(63,4 \%)$ & $98(36,6 \%)$ & 268 \\
\hline
\end{tabular}

El nivel de satisfacción también presentó una relación estadísticamente significativa con la LM $(p=0,028)$.

Existen otras variables relacionadas de manera estadísticamente significativa con la LM, como son: número de hijos, estado civil, talleres de preparación al parto, solicitar ayuda en LM, experiencia personal como la principal fuente de información materno infantil y conocer y haber participado en HL (Tabla 3). 
Tabla 3.- Variables que presentan una relación estadísticamente significativa con la LM.

\begin{tabular}{|c|c|c|c|}
\hline Variables & $\mathbf{n}$ & $\mathbf{L M}$ & $\mathbf{p}$ \\
\hline Número de hijos & & & $<0,001$ \\
\hline 1 & 123 & $31(25,2 \%)$ & \\
\hline 2 & 111 & $49(44,1 \%)$ & \\
\hline 3 & 30 & $15(50 \%)$ & \\
\hline 4 & 1 & $1(100 \%)$ & \\
\hline 5 & 1 & $0(0 \%)$ & \\
\hline 6 & 1 & $0(0 \%)$ & \\
\hline 7 & 2 & $2(100 \%)$ & \\
\hline Estado civil & & & 0,014 \\
\hline Soltera & 75 & $49(65,3 \%)$ & \\
\hline Casada & 190 & $121(63,7 \%)$ & \\
\hline Divorciada & 3 & $0(0 \%)$ & \\
\hline La experiencia personal. & & & 0,004 \\
\hline si & 120 & $65(54,2 \%)$ & \\
\hline no & 148 & $105(70,9 \%)$ & \\
\hline Asiste a los talleres preparación al parto & & & $<0,001$ \\
\hline Si & 190 & $135(71,1 \%)$ & \\
\hline no & 78 & $35(44,9 \%)$ & $\mathbf{P}$ \\
\hline $\mathrm{N}^{\mathrm{o}}$ sesiones asistidas & & & $<0,001$ \\
\hline 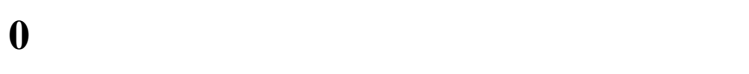 & 78 & $35(44,9 \%)$ & \\
\hline 1 & 2 & $1(50 \%)$ & \\
\hline 2 & 6 & $5(83,3 \%)$ & \\
\hline 3 & 11 & $5(45,5 \%)$ & \\
\hline 4 & 37 & $18(66,7 \%)$ & \\
\hline 5 & 40 & $31(77,5 \%)$ & \\
\hline 6 & 104 & $75(72,1 \%)$ & \\
\hline Busca ayuda en LM & & & $<0,001$ \\
\hline si & 197 & $132(67 \%)$ & \\
\hline no & 71 & $38(53,5 \%)$ & \\
\hline $\begin{array}{l}\text { Conoce la asociación de apoyo a la LM } \\
\text { si }\end{array}$ & 101 & $77(76,2 \%)$ & 0,001 \\
\hline no & 167 & $93(55,7 \%)$ & \\
\hline Asiste a la asociación de apoyo a la LM & & & $<0,001$ \\
\hline 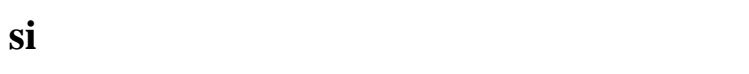 & 22 & $22(100 \%)$ & \\
\hline no & 246 & $148(60,2 \%)$ & \\
\hline
\end{tabular}


El porcentaje de las madres que dan LM aumenta con el número de hijos que tengan. El $71,05 \%$ de las madres que acuden a los talleres de preparación al parto, dan LM a sus hijos y el $100 \%$ de las que acuden a la asociación de apoyo a la lactancia.

Dar el pecho influye en la edad de comienzo de la alimentación complementaria $(p=0,02)$.

La edad de la madre no presenta una relación estadísticamente significativa con la LM $(p=0,848)$.

Tampoco la presentan: tipo de parto, situación laboral, con quien convive; familia, pediatra, matrona e Internet como fuente principal de información materno infantil durante el embarazo; ejercicio físico realizado durante el embarazo, actividad realizada en el postparto con el bebé y haber enviado a su hijo a un centro de educación infantil (Tabla 4).

Tabla 4.- Variables que no presentan una relación estadísticamente significativa con la LM.

\begin{tabular}{|c|c|c|c|}
\hline Variables & $\mathbf{n}$ & $\mathbf{L M}$ & $\mathbf{p}$ \\
\hline Tipo de parto: & & & 0,293 \\
\hline Natural & 196 & $128(65,3 \%)$ & \\
\hline Quirúrgico & 72 & $42(58,3 \%)$ & \\
\hline Situación laboral: & & & 0,5 \\
\hline Empleada & 128 & $86(67,2 \%)$ & \\
\hline Desempleada & 140 & $84(60,0 \%)$ & \\
\hline Con quién convive: & & & 0,157 \\
\hline En pareja & 232 & $148(63,8 \%)$ & \\
\hline Sola & 5 & $2(40 \%)$ & \\
\hline Con sus padres & 16 & $13(81,2 \%)$ & \\
\hline Otra situación & 15 & $7(46,7 \%)$ & \\
\hline $\begin{array}{l}\text { La familia como fuente principal de } \\
\text { información durante el embarazo: }\end{array}$ & & & 0,553 \\
\hline $\mathbf{S i}$ & 124 & $81(65,3 \%)$ & \\
\hline No & 144 & $89(61,8 \%)$ & \\
\hline $\begin{array}{l}\text { El pediatra como fuente principal de } \\
\text { información durante el embarazo: }\end{array}$ & & & 0,23 \\
\hline Si & 63 & $36(57,1 \%)$ & \\
\hline No & 205 & $134(65,4 \%)$ & \\
\hline
\end{tabular}




\begin{tabular}{|c|c|c|c|}
\hline $\begin{array}{l}\text { La matrona como fuente principal de } \\
\text { información durante el embarazo: } \\
\quad \mathrm{Si} \\
\quad \mathrm{No} \\
\text { Internet como fuente principal de } \\
\text { información durante el embarazo: } \\
\quad \mathrm{Si} \\
\quad \mathrm{No}\end{array}$ & $\begin{array}{l}170 \\
98\end{array}$ & $\begin{array}{c}115(67,6 \%) \\
55(56,5 \%) \\
67(70,5 \%) \\
103(59,5 \%)\end{array}$ & 0,060 \\
\hline $\begin{array}{l}\text { Ejercicio físico durante el embarazo: } \\
\text { Si } \\
\text { No } \\
\end{array}$ & $\begin{array}{c}31 \\
237 \\
\end{array}$ & $\begin{array}{c}24(77,4 \%) \\
146(61,6 \%)\end{array}$ & 0,086 \\
\hline $\begin{array}{l}\text { Actividad en el postparto con el bebé: } \\
\text { Si } \\
\text { No }\end{array}$ & $\begin{array}{c}6 \\
262 \\
\end{array}$ & $\begin{array}{c}5(83,3 \%) \\
165(63,0 \%) \\
\end{array}$ & 0,308 \\
\hline $\begin{array}{l}\text { Centro de educación infantil: } \\
\qquad \begin{array}{l}\text { Si } \\
\text { No }\end{array}\end{array}$ & $\begin{array}{l}151 \\
117\end{array}$ & $\begin{array}{l}98(64,9 \%) \\
72(61,5 \%)\end{array}$ & 0,5 \\
\hline
\end{tabular}

Se puede observar que aunque no exista una relación estadísticamente significativa con respecto a con quien convivan las madres, la proporción de mujeres que dan LM es superior para aquellas que viven con su pareja $(63,8 \%)$, con respecto a las que viven solas con su hijo (40\%).

La media de duración de la LM de la muestra es de 3,78 meses (DT 4,7). Las variables: formación académica, talleres de preparación al parto, número de sesiones, nivel de satisfacción, solicitar ayuda sobre LM y conocer y participar en la asociación de apoyo a la lactancia, han presentado una relación estadísticamente significativa con el tiempo de duración de la LM (tabla 5). A mayor formación académica de las madres, mayor duración de la LM.

Tabla 5. Variables que presentan una relación estadísticamente significativa con la duración de la LM.

\begin{tabular}{|c|c|c|c|}
\hline Variables & n & Tiempo de LM & p \\
\hline Formación académica: & & & 0,014 \\
Sin estudios & 13 & 2,1 (DT 2,7) & \\
EGB/ESO & 71 & 3,0 (DT 4,8) & \\
Bachiller/Modulo/FP & 95 & 3,9 (DT 4,6) & \\
Universitarios & 89 & 4,4 (DT 4,9) & \\
\hline Talleres de preparación al parto: & & & 0,029 \\
Si & 190 & 4,2 (DT 4,6) & \\
No & 78 & 2,8 (DT 4,7) & \\
\hline
\end{tabular}




\begin{tabular}{|c|c|c|c|}
\hline$N^{0}$ sesiones asistidas: & 78 & 2,8 (DT 4,7) & 0,002 \\
$\mathbf{0}$ & 2 & 7,5 (DT 10,6) & \\
$\mathbf{1}$ & 6 & 5,5 (DT 4,9) & \\
$\mathbf{2}$ & 11 & 2,5 (DT 3,8) & \\
$\mathbf{3}$ & 37 & 3,7 (DT 4,9) & \\
$\mathbf{4}$ & 40 & 4,4 (DT 4,0) & \\
$\mathbf{5}$ & 104 & 4,2 (DT 4,8) & \\
$\mathbf{6}$ & & & \\
\hline Solicita ayuda en LM: & 71 & 5,6 (DT 4,6) & \\
Si & 197 & 3,1 (DT 4,6) & \\
No & & & 0,004 \\
\hline Conoce la asociación de apoyo a la LM: & 101 & 4,8 (DT 4,8) & \\
Si & 167 & 3,1 (DT 4,5) & \\
No & & & \\
\hline Asiste a la asociación de apoyo a la LM: & 22 & 8,4 (DT 5,1) & \\
Si & 246 & 3,4 (DT 4,4) & \\
No & & & \\
\hline
\end{tabular}

La duración de la LM influye en el mes de inicio de la alimentación complementaria $(p<0,001 ; r=0,32 ; r 2=0,101)$. De la misma manera se ha encontrado relación entre el nivel de satisfacción y la duración de la LM $(p=0,020, r=0,12)$.

Ni la edad de la madre en el momento del parto $(p=0,92, r=0,006)$, ni el número de hijos $(p=0,87)$ presentan relación con la duración de la $L M$. Las demás variables que no presentan una relación estadísticamente significativa con la duración de la LM se exponen en la tabla 6.

Tabla 6.- Variables que no presentan una relación estadísticamente significativa con la duración de la LM.

\begin{tabular}{|c|c|c|c|}
\hline \multicolumn{1}{|c|}{ Variables } & n & Media y DT & p \\
\hline Situación laboral: & & & 0,931 \\
Empleada & 128 & 3,8 (DT 4,3) & \\
Desempleada & 140 & 3,8 (DT 5,0) & \\
\hline Tipo de parto: & & & 0,235 \\
Natural & 196 & 3,9 (DT 4,8) & \\
Quirúrgico & 72 & 3,2 (DT 4,4) & \\
\hline Con quién convive: & & & 0,935 \\
Con su pareja & 232 & 4,0 (DT 4,9) & \\
Sola & 5 & 3,2 (DT 5,2) & \\
\hline
\end{tabular}




\begin{tabular}{|c|c|c|c|}
\hline Con sus padres & 16 & $2,7(\mathrm{DT} 2,5)$ & \\
\hline Otros & 15 & $1,7(\mathrm{DT} 2,2)$ & \\
\hline Ejercicio físico durante el embarazo: & & & 0,17 \\
\hline Sí & 31 & $4,9(\mathrm{DT} 4,8)$ & \\
\hline No & 237 & $3,6(\mathrm{DT} 4,7)$ & \\
\hline Actividad postparto con el bebé: & & & 0,365 \\
\hline Sí & 6 & 5,5 (DT 5,2) & \\
\hline No & 262 & $3,7(\mathrm{DT} 4,7)$ & \\
\hline $\begin{array}{l}\text { La familia como fuente principal de } \\
\text { información durante el embarazo: }\end{array}$ & & & 0,15 \\
\hline $\mathbf{S i}$ & 124 & 3.3 (DT 4.0$)$ & \\
\hline No & 144 & 4.1 (DT 5.2) & \\
\hline $\begin{array}{l}\text { El pediatra como fuente principal de } \\
\text { información durante el embarazo: }\end{array}$ & & & 0,414 \\
\hline Si & & & \\
\hline No & 63 & $4,2(\mathrm{DT} 5,2)$ & \\
\hline $\begin{array}{l}\text { La matrona como fuente principal de } \\
\text { información durante el embarazo: }\end{array}$ & 205 & 3,6 (DT 4,5) & 0,833 \\
\hline $\mathbf{S i}$ & 170 & 3,7 (DT 4,3) & \\
\hline No & 98 & $3,8($ DT 5,2$)$ & \\
\hline $\begin{array}{l}\text { Internet como fuente principal de } \\
\text { información durante el embarazo: }\end{array}$ & & & 0,30 \\
\hline $\mathbf{S i}$ & 95 & $4,4(\mathrm{DT} 4,7)$ & \\
\hline No & 173 & 3,5 (DT 4,7) & \\
\hline Centro de educación infantil: & & & 0,59 \\
\hline Sí & 151 & $3,6(\mathrm{DT} 4,3)$ & \\
\hline No & 117 & 3,9 (DT 5,1) & \\
\hline
\end{tabular}

En el análisis multivariante mediante la regresión logística binaria para la LM como variable dependiente, se obtuvo que las que presentaban relación eran: pedir ayuda en LM $(p<0,001)$, inicio de la alimentación complementaria $(p=0,02)$ y el nivel de satisfacción que aunque presenta una relación al borde de la significación estadística, permaneció en el modelo por ser relevante para la explicación de la $\operatorname{LM}(p=0,057)$.

Al realizar la regresión lineal múltiple con la duración de la LM como variable dependiente, se obtuvo que las variables que presentan relación son: pedir ayuda en LM $(p=0,02)$, nivel de satisfacción $(p=0,025)$, comienzo de la alimentación complementaria $(p<0,001)$ y haber asistido a la asociación de apoyo a la lactancia $(p=0,005)$. También se incluyó en el modelo la formación académica $(p=0,096)$ : su presencia mejoraba la explicación de la variable dependiente. 


\section{DISCUSIÓN}

A pesar de la labor de la promoción en Atención Primaria de la LM, la prevalencia y duración de la misma son inferiores a las recomendaciones de la OMS ${ }^{(3)}$.

En Huelva el porcentaje de inicio del $64,3 \%$ es inferior al que muestran otros estudios a nivel nacional, como es el caso del $82,5 \%$ de un estudio realizado en Aragón ${ }^{(15)}$, $80,5 \%$ en León ${ }^{(16)}$ o el $81,2 \%$ en Palma de Mallorca ${ }^{(17)}$.

Los resultados de prevalencia de la LM son inferiores a las observadas en países europeos (en torno al $90 \%$ al inicio en Alemania y del $50 \%$ a los tres meses del nacimiento en Grecia) ${ }^{(18-19)}$.

Según los datos obtenidos y coincidiendo con los resultados de otros estudios, se observa cómo las madres que tienen el apoyo por parte de sus parejas, obtienen beneficios que en este caso se reflejan en el hecho de dar el pecho a sus hijos y los meses dedicados a la misma ${ }^{(20)}$.

La formación académica de las madres ha sido un factor que ha destacado en los resultados, ya que es favorable tanto para la prevalencia como duración de la LM. Existe un estudio que compara estos datos desde finales de los 50 hasta el 2002 y obtiene como resultados que las mujeres con estudios superiores han ido aumentando la duración de LM a lo largo del tiempo hasta nuestros días ${ }^{(21)}$.

Son muchos los estudios dedicados a la importancia de los talleres de preparación para el parto para la promoción de la LM. Los resultados que se han obtenido coinciden fielmente con ellos en que aquellas madres que acudan a estos talleres y las que lo hagan con el mayor número de veces tendrán una repercusión directa en la prevalencia y duración de la $\mathrm{LM}^{(18)}$.

Pero también hay que tener en cuenta que la transmisión de conocimientos sobre la salud ya no se hace sólo entre el profesional de la salud y el paciente. Cada vez más son los padres que consultan libros y en Internet en relación a estos temas ${ }^{(22)}$.

Se debe incidir en el respeto y fomento de las decisiones tomadas por las mujeres en relación a la lactancia. Es importante reforzar las medidas de información y promoción de la leche materna en los centros de Salud, centros hospitalarios y pediatras para que sean las madres las que adopten su propia decisión. De una información adecuada y del respeto va a depender en gran medida la satisfacción que estas mujeres experimenten sobre la información recibida y se ha comprobado que las mujeres satisfechas en este sentido dan más el pecho a sus hijos y durante más meses que las que no lo están ${ }^{(23)}$.

Tanto las madres que conocen el grupo de apoyo a la lactancia como las que han acudido a alguna de sus reuniones, destacan positivamente en la prevalencia y duración de la LM. Es necesario potenciar más estas agrupaciones así como reforzar un inicio de la alimentación complementaria a los 6 meses y no antes ${ }^{(10)}$.

\section{CONCLUSIONES}

La situación en Andalucía respecto a la LM, y más aún en Huelva, dista mucho de lo recomendado por los organismos internacionales. 
La mayor parte de las mujeres que la inician, la abandonan de manera muy temprana, en los primeros meses de vida del bebé.

Los factores que más influyen en la LM están relacionados con la educación materna, tanto a nivel académico como de educación sanitaria, por lo que los datos muestran que queda mucho trabajo por hacer.

\section{REFERENCIAS}

(1) Organización Mundial de la Salud. Lactancia materna: Salud de la madre, el recién nacido, del niño y del adolescente. 2015.

(2) Massó Guijarro E. Lactancia materna y revolución, o la teta como insumisión biocultural: calostro, cuerpo y cuidado. Dilemata. 2013; 11:169-206.

(3) Organización Mundial de la Salud. 10 datos sobre la lactancia materna. 2013.

(4) Rius J M, Ortuño J, Rivas C, Maravall M, Calzado MA, López A et al. Factores asociados al abandono precoz de la lactancia materna en una región del este de España. An Pediatr. 2014; 80(1):6-15.

(5) Capotorto ML, Irumberri A. Doulas: compañía emocional como labor. I Jornadas de Género y Diversidad Sexual (GEDIS)(La Plata, 2014). 2014.

(6) Lavender T, Richens Y, Milan SJ, Smyth R, Dowswell T. Telephone support for women during pregnancy and the first six weeks postpartum. Cochrane Libr. 2013.

(7) Gámez Requena JJ, Márquez Feu T, Gómez Gómez FJ, Sánchez García E. Efecto de una campaña de promoción de la lactancia materna en la provincia de Málaga en mujeres cuyos partos finalizaron con cesárea. Aten Primaria. 2004; 33:503-506.

(8) Instituto Nacional de Estadística. Tipo de lactancia por clase social basada en la ocupación de la persona de referencia duración. 2011-2012.

(9) Camacho IG, Duque NR. Estudio de Prevalencia de Lactancia Materna en la isla de La Palma. ENE, Rev Enf. 2012; 4(1).

(10) Botello-Cabrera MT, Marín H, Vera M, Parrilla-Rodríguez AM. Factores asociados al inicio, tipo y duración de la lactancia materna entre participantes y no participantes a charla prenatal de educación en lactancia materna de una institución hospitalaria. Puerto Rico Health Sci J. 2014; 18(3).

(11) Cebrián DM, Santana RM, Villanueva EG, Santana PS. Factores relacionados con el abandono de la lactancia materna. An Pediatr. 2002; 56(2):144-150.

(12) Gámez Requena J, Márquez Feu T, Gómez Gómez F, Sánchez García E. Efecto de una campaña de promoción de la lactancia materna en la provincia de Málaga en mujeres cuyos partos finalizaron con cesárea. Aten Primaria. 2004; 33(9):503-506.

(13) Casanova MG, Casanova SG, Juan MP, Mariscal ER, Esquius NP. Lactancia materna: ¿Puede el personal sanitario influir positivamente en su duración?. Aten Primaria. 2005; 35(6):295-300.

(14) Olmo Navarro JE, Molina-Ruano MD, López Sánchez-Sánchez R, Bermúdez Ruiz MI, Castaño Molina MA. Duración de la lactancia materna en el noroeste murciano. Matronas Prof. 2008; 9(2):12-16.

(15) Andrés LC, Villagrasa MS, Sauras MÁ, Velillas JL, Martínez GR, Calina GC. Prevalencia de la lactancia materna durante el primer año de vida en Aragón. Estudio CALINA. An Pediatr. 2013; 79(5):312-318.

(16) Rodríguez MM, Rilo JN, Fernández EB, Arias IC, Fernández MR, Casado AP. Prevalencia y duración de la lactancia materna. Influencia sobre el peso y la morbilidad. Nutr Hosp. 2009; 24(2):213-217.

(17) Moll Pons JM, Prieto Valle JM, Sánchez Martínez A, López León MP, Arana Galán JM, Frontera Juan G. Prevalencia de la lactancia materna en el sector de salud 
del Hospital «Son LLàtzer» de Palma de Mallorca. Acta Pediatr Esp. 2012; 70(5):186194.

(18) Kohlhuber M, Rebhan B, Schwegler U, Koletzko B, Fromme H. Breastfeeding rates and duration in Germany: a Bavarian cohort study. Br J Nutr. 2008; 99(05):11271132.

(19) Bakoula C, Veltsista A, Prezerakou A, Moustaki M, Fretzayas A, Nicolaidou P. Working mothers breastfeed babies more than housewives. Acta Paediatr. 2007; 96(4):510-515.

(20) Ramírez MA. Padres y desarrollo de los hijos: Prácticas de crianza. Estudios pedagógicos (Valdivia) 2005; 31(2):167-177.

(21) Colodro Conde L, Sánchez Romera JF, Tornero Gómez MJ, Pérez Riquelme F, Polo Tomás M, Ordoñana JR. Relationship Between Level of Education and Breastfeeding Duration Depends on Social Context Breastfeeding Trends Over a 40Year Period in Spain. J Hum Lactation. 2011; 27(3):272-278.

(22) González Rodríguez MP, González de Dios J, Buñuel Álvarez JC. La información para padres en Internet y Evidencias en Pediatría. Evid Pediatr. 2012; 8(3).

(23) Aguilar Cordero MJ, Sáez Martín I, Menor Rodríguez MJ, Mur Villar N, Expósito Ruiz M, Hervás Pérez A et al. Valoración del nivel de satisfacción en un grupo de mujeres de Granada sobre atención al parto, acompañamiento y duración de la lactancia. Nutr Hosp. 2013; 28(3):920-926.

Recibido: 19 de abril 2015; Aceptado: 9 de julio 2015

ISSN 1695-6141 